\title{
Atrial fibrillation after electrical cardioversion in elderly patients: a role for arterial stiffness? Results from a preliminary study
}

\author{
Stefano Fumagalli ${ }^{1}$ (D) Ilaria Giannini ${ }^{1} \cdot$ Simone Pupo $^{1} \cdot$ Francesca Agostini $^{2} \cdot$ \\ Serena Boni ${ }^{1} \cdot$ Anna T. Roberts ${ }^{1} \cdot$ Debbie Gabbai $^{1} \cdot$ Claudia Di Serio $^{1}$. \\ Luciano Gabbani $^{1} \cdot$ Francesca Tarantini $^{1} \cdot$ Niccolò Marchionni $^{1}$
}

Received: 27 May 2016/Accepted: 9 August 2016/Published online: 27 August 2016

(c) The Author(s) 2016. This article is published with open access at Springerlink.com

\begin{abstract}
Background and aims Atrial fibrillation (AF) is the most frequent arrhythmia of the elderly, and electrical cardioversion (ECV) is a common procedure, although incidence of recurrences remains high. We evaluated the possible association between arterial stiffness (AS) and the persistence or recurrence of $\mathrm{AF}$ in elderly patients after ECV.

Methods We enrolled all subjects undergoing ECV over a 9-month period. AS was evaluated with the cardio-ankle vascular index (CAVI). Patients were then visited at follow-up (on average at 6 months).

Results Thirty-one patients (age $78 \pm 7$ years; men $67.7 \%$; $\mathrm{CHA}_{2} \mathrm{DS}_{2}$-VASc $4.1 \pm 1.6$; AF length $>2$ months $51.6 \%$; CAVI $9.9 \pm 1.6$ ) underwent ECV. At follow-up, sinus rhythm was recorded in $16(51.6 \%)$ patients. At multivariate analysis, the presence of $\mathrm{AF}$ was directly associated with $\mathrm{CHA}_{2} \mathrm{DS}_{2}$-VASc score and CAVI. Amiodarone therapy reduced the risk of relapsed AF.

Conclusions In elderly AF patients treated with ECV, AS at baseline seems to predict AF at follow-up.
\end{abstract}

Keywords Arterial stiffness - Atrial fibrillation - CAVI . $\mathrm{CHA}_{2} \mathrm{DS}_{2}$-VASc $\cdot$ Elderly $\cdot$ Electrical cardioversion

Stefano Fumagalli

fumadue@tin.it

1 Geriatric Intensive Care Unit, Experimental and Clinical Medicine Department, University of Florence and AOU Careggi, Florence, Italy

2 School of Nursing, University of Florence, Florence, Italy

\section{Introduction}

Atrial fibrillation (AF) is the most common sustained rhythm disorder of the elderly [1]. Arterial stiffness (AS) increases with age and predicts coronary heart disease, stroke and mortality [2]. In a previous pilot study on AF patients, we found that AS could represent an important factor associated with left atrium (LA) remodeling [3]. In the present study, we evaluated whether AS has a role in sinus rhythm maintenance in elderly subjects undergoing elective electrical cardioversion (ECV) of persistent AF. We also examined concentrations of interleukin-6 (IL-6), which are associated with AS in healthy adults [4] and early $\mathrm{AF}$ recurrences [5].

\section{Methods}

\section{Patients and procedures}

We enrolled all patients admitted to our day-hospital for ECV between March and December 2015. At baseline, all patients underwent assessment of physical and neuro-cognitive function with the short physical performance battery (SPPB) and the mini-mental state examination (MMSE). Depressive symptoms were evaluated with the geriatric depression scale (GDS). Venous IL-6 concentrations were determined in 27 out of $31(87.1 \%)$ patients using commercially available ELISA kits (R\&D Systems, Inc.; Minneapolis, MN, USA). ECV was performed using a biphasic defibrillator, after a 4-week period of effective oral anticoagulation [6].

The cardio-ankle vascular index (CAVI) [3, 7], a measure of AS, independent from instantaneous systolic and pulse pressure, was evaluated immediately before 
discharge using VaSera VS-1500N (Fukuda Denshi, Japan). During the continuous recording of EKG and heart sounds, right and left upper and lower extremity arterial pressure was obtained with the oscillometric method. Pulse wave velocity (PWV) was calculated dividing the distance from the aortic valve to the ankle by the sum of two time intervals (1. aortic valve closing sound-notch of the brachial pulse wave; 2 . rise of the brachial pulse wave-rise of the ankle pulse wave) [7]. CAVI was then computed using the following equation:

$\mathrm{CAVI}=a \times\left[(2 \rho / \Delta P) \times \ln \left(P_{\mathrm{s}} / P_{\mathrm{d}}\right) \times \mathrm{PWV}^{2}\right]+b$

where $P_{\mathrm{s}} / P_{\mathrm{d}}$ are systolic/diastolic pressures, $\Delta P$ is " $P_{\mathrm{s}}-$ $P_{\mathrm{d}} ", \rho$ is blood density, and $a / b$ are constants [7].

At follow-up, arterial blood pressure, EKG, SPPB, MMSE, GDS and main clinical events were recorded in all cases.

\section{Statistical analysis}

IBM SPSS for Windows (version 23) was used for statistical analysis. Continuous and categorical variables are expressed as mean $\pm \mathrm{SD}$ and numbers with percentages, respectively. Linear regression analysis described the relation between continuous variables. Student's $t$ test and analysis of variance-or the related nonparametric tests-were used to compare continuous variables between groups. The association between categorical variables was evaluated with Chisquare test. Logistic regression analysis models identified clinical predictors of sinus rhythm at follow-up. A two-tailed $p$ value $<0.05$ was considered statistically significant.

\section{Results}

\section{Study population}

Thirty-eight patients underwent elective ECV. We excluded 7 subjects ( $18.4 \%$ of the whole population) because of ECV of an arrhythmia relapse $(N=3)$, age $<55$ years, severe sinus node dysfunction, logistic reasons and refusal to participate $(N=1$ each). Characteristics of the 31 patients included in the study are reported in Table 1.

Mean age was 78 years (33rd-66th percentile: $76-80$ years). On the whole, neuro-cognitive function was preserved and prevalence of disability low.

Hypertension emerged as the most important risk factor for AF. Arrhythmia length was $>2$ months in 16 subjects $(51.6 \%) . \mathrm{CHA}_{2} \mathrm{DS}_{2}$-VASc score and AS were high. Only 7 patients $(22.6 \%)$ showed CAVI values $<9$ [7].

Most patients received antagonists of the renin-angiotensin system and beta-blockers; amiodarone was the most frequently used antiarrhythmic drug (Table 1).

\section{AF predictors at follow-up}

ECV was effective in $90.3 \%$ of patients $(N=28 / 31)$. At the follow-up evaluation (mean length: 179 days, 33rd66th percentile: 104-252 days), sinus rhythm was observed in $51.6 \%$ of population $(N=16)$.

At follow-up, patients presenting AF tended to be older, but no differences were observed according to gender, neuro-cognitive and functional profile, depressive symptoms and history of CHF. Prevalence of diabetes, $\mathrm{CHA}_{2-}$ $\mathrm{DS}_{2}$-VASc score, baseline systolic arterial pressure, AS and $\mathrm{AF}$ duration were all significantly higher in those with AF at follow-up (Table 1; Fig. 1). Regarding drugs, only amiodarone was associated with a higher persistence of sinus rhythm. Subjects with AF showed higher IL-6 concentrations (Fig. 1); a pattern between higher levels of the cytokine and age, diabetes, $\mathrm{AS}, \mathrm{CHA}_{2} \mathrm{DS}_{2}$-VASc score, anti-gout therapy and a poor SPPB performance also emerged.

Multivariate logistic regression analysis demonstrated that the presence of $\mathrm{AF}$ at follow-up visit was directly correlated with the $\mathrm{CHA}_{2} \mathrm{DS}_{2}$-VASc score and AS, while amiodarone therapy maintained an independent protective association (Table 2).

\section{Discussion}

The results of the present study demonstrate that ECV is extremely effective also in elderly patients, with a success rate as high as $90 \%$. However, relapse is frequent; at follow-up, about $50 \%$ of our population showed AF. The presence of the arrhythmia, due to new episodes and to previous ECV failures, was inversely associated with amiodarone therapy and directly related to cardio-embolic risk and to AS, as expressed by the $\mathrm{CHA}_{2} \mathrm{DS}_{2}$-VASc score and CAVI, respectively.

The efficacy of amiodarone in preventing AF recurrences has already been demonstrated. The "Canadian Trial of Atrial Fibrillation Investigators" showed that the incidence of arrhythmia relapses with amiodarone was lower than that observed with sotalol or propafenone, but the rate of adverse events was also higher [8]. More recently, a study, conducted in the Department of Veterans Affairs National Health Care System on subjects with a newly diagnosed AF, found that amiodarone use was not associated with increased mortality after adjustment for age, gender, the presence of CHF, renal function and use of beta-blockers and warfarin [9].

In patients undergoing electrical or pharmacological cardioversion, the $\mathrm{CHA}_{2} \mathrm{DS}_{2}$-VASc score emerged as a significant predictor of early recurrence of AF [10]. Similarly, in the "Leipzig Heart Center AF Ablation Registry," 
Table 1 Main characteristics in all patients and by $\mathrm{AF}$ at followup

\begin{tabular}{|c|c|c|c|c|}
\hline & \multirow[t]{2}{*}{ All patients } & \multicolumn{2}{|c|}{$\mathrm{AF}$ at follow-up } & \multirow[t]{2}{*}{$p$} \\
\hline & & No & Yes & \\
\hline \multicolumn{5}{|l|}{ Continuous variables } \\
\hline Age (years) & $78 \pm 7$ & $74 \pm 8$ & $81 \pm 4$ & 0.005 \\
\hline Height $(\mathrm{cm})$ & $170 \pm 9$ & $170 \pm 8$ & $171 \pm 9$ & 0.593 \\
\hline Weight (Kg) & $74 \pm 14$ & $75 \pm 13$ & $73 \pm 15$ & 0.637 \\
\hline MMSE (score) & $27.6 \pm 3.4$ & $28.4 \pm 1.5$ & $26.9 \pm 4.4$ & 0.243 \\
\hline GDS (score) & $3.4 \pm 1.8$ & $3.4 \pm 1.6$ & $3.4 \pm 2.3$ & 0.980 \\
\hline SPPB (total score) & $9.6 \pm 2.2$ & $9.8 \pm 2.4$ & $9.4 \pm 2.0$ & 0.424 \\
\hline $\mathrm{CHA}_{2} \mathrm{DS}_{2}$-VASc (score) & $4.1 \pm 1.6$ & $3.3 \pm 1.4$ & $5.0 \pm 1.4$ & 0.003 \\
\hline HR (bpm) & $74 \pm 14$ & $73 \pm 15$ & $74 \pm 14$ & 0.803 \\
\hline SAP (mmHg) & $134 \pm 21$ & $127 \pm 20$ & $141 \pm 20$ & 0.036 \\
\hline DAP (mmHg) & $77 \pm 11$ & $75 \pm 10$ & $80 \pm 11$ & 0.178 \\
\hline Left atrium diameter (mm) & $52 \pm 4$ & $52 \pm 4$ & $52 \pm 5$ & 0.709 \\
\hline IVS thickness (mm) & $10 \pm 1$ & $10 \pm 1$ & $10 \pm 1$ & 0.839 \\
\hline $\operatorname{LVEDD}(\mathrm{mm})$ & $50 \pm 7$ & $51 \pm 6$ & $49 \pm 7$ & 0.308 \\
\hline $\operatorname{LVEF}(\%)$ & $61 \pm 9$ & $61 \pm 9$ & $61 \pm 8$ & 0.980 \\
\hline \multicolumn{5}{|l|}{ Categorical variables $(N, \%)$} \\
\hline Men & $21(67.7)$ & $11(68.8)$ & $12(66.7)$ & 1.000 \\
\hline Living alone & $6(19.4)$ & $2(12.5)$ & $4(26.7)$ & 0.394 \\
\hline Smokers (present/past) & $16(51.6)$ & $8(50.0)$ & $8(53.3)$ & 1.000 \\
\hline Wine ( $\geq 1$ glass/day) & $19(61.3)$ & $10(62.5)$ & $9(60.0)$ & 1.000 \\
\hline $\mathrm{CAD}$ & $9(29.1)$ & $4(25.0)$ & $5(33.3)$ & 0.704 \\
\hline $\mathrm{CHF}$ & $15(48.4)$ & $7(43.8)$ & $8(53.3)$ & 0.724 \\
\hline Chronic renal failure & $3(9.7)$ & $1(6.3)$ & $2(13.3)$ & 0.600 \\
\hline COPD & $3(9.7)$ & $0(0)$ & $3(20.0)$ & 0.101 \\
\hline CVD & $4(12.9)$ & $2(12.5)$ & $2(13.3)$ & 1.000 \\
\hline Diabetes & $8(25.8)$ & $1(6.3)$ & 7 (46.7) & 0.015 \\
\hline Dyslipidemia & $14(45.2)$ & $5(31.3)$ & $9(60.0)$ & 0.156 \\
\hline Hypertension & $27(87.1)$ & $12(75.0)$ & $15(100)$ & 0.101 \\
\hline $\mathrm{PAD} /$ aortic aneurism & $5(16.2)$ & $1(6.3)$ & $4(26.7)$ & 0.172 \\
\hline Thyroid dysfunction & $9(29.0)$ & $4(25.0)$ & $5(33.3)$ & 0.704 \\
\hline AF length $>2$ months & $16(51.6)$ & $4(25.0)$ & $12(80.0)$ & 0.004 \\
\hline ACE-I/ARBs & $27(87.1)$ & $14(87.5)$ & $13(86.7)$ & 1.000 \\
\hline Beta-blockers & $22(71.0)$ & $13(81.3)$ & $9(60.0)$ & 0.252 \\
\hline Statins & $15(48.4)$ & $7(43.8)$ & $8(53.3)$ & 0.724 \\
\hline Digitalis & $13(41.9)$ & $7(43.8)$ & $6(40.0)$ & 1.000 \\
\hline Amiodarone & $14(45.2)$ & $11(68.8)$ & $3(20.0)$ & 0.011 \\
\hline Class IC AADs & $4(12.9 \%)$ & $1(6.3)$ & $3(20.0)$ & 0.333 \\
\hline OAT—warfarin & $21(67.7)$ & $10(62.5)$ & $11(73.3)$ & 0.704 \\
\hline OAT-NOACs & $10(32.3)$ & $6(37.5)$ & $4(26.7)$ & \\
\hline
\end{tabular}

$A R B$ s Angiotensin receptor blockers, $C A D$ coronary artery disease, $C H F$ chronic heart failure, Class IC $A A D s$ class I C antiarrhythmic drugs, $C O P D$ chronic obstructive pulmonary disease, $C V D$ cerebrovascular disease, GDS geriatric depression scale, $H R$ heart rate, IVS interventricular septum, $L V E D D$ left ventricular end-diastolic diameter, $L V E F$ left ventricular ejection fraction, $M M S E$ mini-mental state examination, $O A T$ oral anticoagulant therapy, NOACs non-VKA oral anticoagulants, PAD/Aortic Aneurism peripheral artery disease/aortic aneurism, SAP/DAP systolic/diastolic arterial pressure, SPPB short physical performance battery, Wine wine consumption or equivalent for other alcoholic beverages 

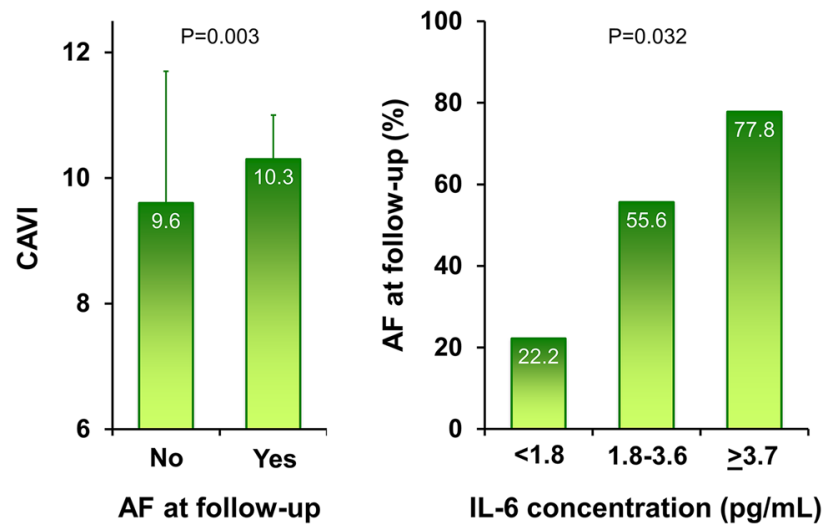

Fig. 1 Arterial stiffness at baseline, measured with cardio-ankle vascular index (CAVI), by the presence of AF at follow-up (left panel) and proportion of patients with $\mathrm{AF}$ at follow-up by tertiles of interleukin-6 (IL-6) concentration (right panel). Because right and left CAVI values were not different $(p=0.872)$, we reported the left ones for their higher statistical association with the endpoint variable

the $\mathrm{CHA}_{2} \mathrm{DS}_{2}$-VASc score was associated with both early and late relapses of the arrhythmia [11]. Hypertension, diabetes and $\mathrm{CHF}$, through their action on inflammation, oxidative stress and atrial fibrosis, could represent a link between the scores of cardio-embolic risk and AF recurrence [11]. Confirming this hypothesis, in our population, we found an inverse association between IL-6 concentration and the prevalence of sinus rhythm at the follow-up. The $\mathrm{CHA}_{2} \mathrm{DS}_{2}$-VASc score seems useful to identify $\mathrm{AF}$ patients with a higher overall risk of events. At this regard, in subjects with a mean age of 73.9 years, during a 2-year follow-up, scoring 9, when compared to 0 , was associated with a three times higher risk to be admitted in hospital for cardiovascular causes [12].

Indeed, the most interesting finding of our preliminary experience was the direct association between AS and the presence of arrhythmia at follow-up. For each one-unit increase in CAVI, the risk of finding $\mathrm{AF}$ at the control visit was 2.31 times higher. Few previous reports, obtained with surrogate measures, enforced our results. After 4.9 years of follow-up, among patients with hypertension and left ventricular hypertrophy enrolled in the "Losartan intervention for endpoint (LIFE) reduction in hypertension study," the incidence of a first episode of the arrhythmia was $4.0 \%$, with a robust, significant, relation with pulse pressure, a marker of AS [13]. Among the Framingham Heart Study participants aged $\geq 35$ years, cumulative 20-year AF incidence rates were 5.6 and $23.3 \%$ for pulse pressure values $\leq 40$ and $>61 \mathrm{mmHg}$, respectively. Hence, AS could play a role on AF-related mechanisms also in community-based cohorts [14].

Our results are the first to show that in an elderly population undergoing $\mathrm{ECV}$ of persistent $\mathrm{AF}$, the presence of arrhythmia at follow-up, more often a recurrence, is directly related to arterial properties. A possible explanation could be represented by the association between CAVI and left atrium diameter, which is independent of left ventricular thickness [3]. Once again, low-grade inflammation could be the link between aortic stiffness and left atrium dilation [15].

The small number of patients is the major limitation of the study. IL-6 concentration was not measured in all subjects. This fact, coupled with the collinear increase in age, diabetes, AS and the $\mathrm{CHA}_{2} \mathrm{DS}_{2}$-VASc score, prevented us from adjusting our models by IL-6 values. However, this is a preliminary, pilot study. The results we obtained are biologically and clinically plausible and could represent the starting point for further, more detailed investigations.

In conclusion, in an elderly AF population, the occurrence of arrhythmia at follow-up seems to be related not only to $\mathrm{CHA}_{2} \mathrm{DS}_{2}$-VASc score and the use of amiodarone, but also to AS. Hence, the modulation of vascular properties could represent a possible target to reduce AF burden and its complications in aged, at-risk subjects.
Table 2 Multivariate predictors of the presence of $\mathrm{AF}$ at follow-up visit

\begin{tabular}{lcll}
\hline & $\beta \pm$ e.s. & OR $(95 \%$ CI $)$ & $p$ \\
\hline CHA $_{2}$ DS $_{2}$-VASc $(\Delta$ point $)$ & $0.97 \pm 0.49$ & $2.65(1.01-6.94)$ & 0.048 \\
CAVI $(\Delta$ unit) & $0.84 \pm 0.42$ & $2.31(1.01-5.25)$ & 0.046 \\
Amiodarone (yes vs. no) & $-2.91 \pm 1.30$ & $0.05(0.01-0.70)$ & 0.025 \\
Constant & $-23.33 \pm 10.54$ & $/$ & 0.027 \\
\hline
\end{tabular}

Results of the multivariate logistic regression analysis (overall predictivity $=83.7 \%$ )

Variables excluded from the model: baseline systolic arterial pressure $(p=0.101)$; AF length $>2$ months $(p=0.429)$

$\Delta$ change in the dependent variable per unitary change in the independent variable, CAVI arterial stiffness assessed with the cardio-ankle vascular index, obtained through arterial pressure measures at the left arm and ankle, $O R$ odds ratio 


\section{Compliance with ethical standards}

Conflict of interest No conflict of interest to declare.

Human and animal rights All procedures were performed in accordance with the principles of the Declaration of Helsinki and approved by the appropriate institutional review committee.

Informed consent All subjects gave their informed consent to participate in the study.

Open Access This article is distributed under the terms of the Creative Commons Attribution 4.0 International License (http://crea tivecommons.org/licenses/by/4.0/), which permits unrestricted use, distribution, and reproduction in any medium, provided you give appropriate credit to the original author(s) and the source, provide a link to the Creative Commons license, and indicate if changes were made.

\section{References}

1. Mozaffarian D, Benjamin EJ, Go AS et al (2016) Heart disease and stroke statistics-2016 update: a report from the American Heart Association. Circulation 133:e38-e360

2. Cavalcante JL, Lima JA, Redheuil A et al (2011) Aortic stiffness: current understanding and future directions. J Am Coll Cardiol 57:1511-1522

3. Fumagalli S, Gabbai D, Nreu B et al (2014) Age, left atrial dimension and arterial stiffness after external cardioversion of atrial fibrillation. A vascular component in arrhythmia maintenance? Results from a preliminary study. Aging Clin Exp Res 26:327-330

4. van Bussel BC, Schouten F, Henry RM et al (2011) Endothelial dysfunction and low-grade inflammation are associated with greater arterial stiffness over a 6-year period. Hypertension 58:588-595

5. Smit MD, Maass AH, De Jong AM et al (2012) Role of inflammation in early atrial fibrillation recurrence. Europace $14: 810-817$
6. Camm AJ, Kirchhof P, Lip GY et al (2010) Guidelines for the management of atrial fibrillation: the task force for the management of atrial fibrillation of the European Society of Cardiology (ESC). Eur Heart J 31:2369-2429

7. Shirai K, Hiruta N, Song M et al (2011) Cardio-ankle vascular index (CAVI) as a novel indicator of arterial stiffness: theory, evidence and perspectives. J Atheroscler Thromb 18:924-938

8. Roy D, Talajic M, Dorian P et al (2000) Amiodarone to prevent recurrence of atrial fibrillation. Canadian trial of atrial fibrillation investigators. N Engl J Med 342:913-920

9. Ullal AJ, Than CT, Fan J et al (2015) Amiodarone and risk of death in contemporary patients with atrial fibrillation: findings from the retrospective evaluation and assessment of therapies in AF study. Am Heart J 170:1033-1041

10. Falsetti L, Viticchi G, Tarquinio N et al (2014) CHA2DS2-VASc in the prediction of early atrial fibrillation relapses after electrical or pharmacological cardioversion. J Cardiovasc Med (Hagerstown) 15:636-641

11. Kornej J, Hindricks G, Kosiuk J et al (2014) Comparison of $\mathrm{CHADS}_{2}, \mathrm{R}_{2} \mathrm{CHADS}_{2}$, and $\mathrm{CHA}_{2} \mathrm{DS}_{2}$-VASc scores for the prediction of rhythm outcomes after catheter ablation of atrial fibrillation: the Leipzig Heart Center AF ablation registry. Circ Arrhythm Electrophysiol 7:281-287

12. Naccarelli GV, Panaccio MP, Cummins G et al (2012) $\mathrm{CHADS}_{2}$ and $\mathrm{CHA}_{2} \mathrm{DS}_{2}$-VASc risk factors to predict first cardiovascular hospitalization among atrial fibrillation/atrial flutter patients. Am J Cardiol 109:1526-1533

13. Larstorp AC, Ariansen I, Gjesdal K et al (2012) Association of pulse pressure with new-onset atrial fibrillation in patients with hypertension and left ventricular hypertrophy: the Losartan intervention for endpoint (LIFE) reduction in hypertension study. Hypertension 60:347-353

14. Mitchell GF, Vasan RS, Keyes MJ et al (2007) Pulse pressure and risk of new-onset atrial fibrillation. JAMA 297:709-715

15. Paulus WJ, Tschope C (2013) A novel paradigm for heart failure with preserved ejection fraction: comorbidities drive myocardial dysfunction and remodeling through coronary microvascular endothelial inflammation. J Am Coll Cardiol 62:263-271 